\title{
Limits on isotropic Lorentz violation in QED from collider physics
}

\author{
Michael A. Hohensee, ${ }^{1}$ Ralf Lehnert, ${ }^{2,3}$ David F. Phillips, ${ }^{4}$ and Ronald L. Walsworth ${ }^{1,4}$ \\ ${ }^{1}$ Department of Physics, Harvard University, Cambridge, Massachusetts, 02138, USA \\ ${ }^{2}$ Max-Planck-Institut für Physik, Föhringer Ring 6, 80805 München, Germany \\ ${ }^{3}$ Instituto de Ciencias Nucleares, Universidad Nacional Autónoma de México, A. Postal 70-543, 04510 México D.F., Mexico \\ ${ }^{4}$ Harvard-Smithsonian Center for Astrophysics, Cambridge, Massachusetts 02138, USA
}

(Dated: November 2, 2018)

\begin{abstract}
We consider the possibility that Lorentz violation can generate differences between the limiting velocities of light and charged matter. Such effects would lead to efficient vacuum Cherenkov radiation or rapid photon decay. The absence of such effects for $104.5 \mathrm{GeV}$ electrons at the Large Electron Positron collider and for $300 \mathrm{GeV}$ photons at the Tevatron therefore constrains this type of Lorentz breakdown. Within the context of the standard-model extension, these ideas imply an experimental bound at the level of $-5.8 \times 10^{-12} \leq \tilde{\kappa}_{\mathrm{tr}}-(4 / 3) c_{e}^{00} \leq 1.2 \times 10^{-11}$ tightening existing laboratory measurements by $3-4$ orders of magnitude. Prospects for further improvements with terrestrial and astrophysical methods are discussed.
\end{abstract}

PACS numbers: 11.30.Cp, 12.20.-m, 41.60.Bq, 29.20.-c

\section{INTRODUCTION}

Established physics is successfully described by two distinct theories: general relativity (GR) and the standard model (SM) of particle physics. These two theories are commonly believed to arise as the low-energy limit of a more fundamental Planck-scale framework that consistently merges gravity and quantum mechanics. Since direct measurements at this scale are presently impractical, experimental research in this field relies largely on ultrahigh-precision searches for Planck-suppressed effects at attainable energies.

One candidate effect within this context is a minute breakdown of Lorentz invariance [1]. Lorentz symmetry represents a cornerstone of both GR and the SM, so that any observed deviation from this symmetry would imply new physics. A number of theoretical approaches to underlying physics, such as strings [2], noncommutative field theories [3], cosmologically varying fields [4], quantum gravity [5], random-dynamics models [6], multiverses [7], brane-world scenarios [8], and massive gravity 9], are known to accommodate small violations of Lorentz invariance at low energies. Searches for such violations are also motivated by the seemingly fundamental character of Lorentz symmetry: it should be buttressed as firmly as possible by experimental evidence.

At currently attainable energies, Lorentz-violating effects are expected to be described by an effective field theory [10]. The standard-model extension (SME) provides the general framework in this context [11, 12], containing both GR and the SM as limiting cases. The additional Lagrangian terms of the SME include all operators for Lorentz violation that are scalars under coordinate changes. The SME has already provided the basis for the analysis of numerous experimental searches for Lorentz breakdown [13], including ones with photons [14, 15, 16, 17], electrons [18, 19, 20], protons and neutrons [21, 22, 23, 24, 25], mesons [26], muons [27], neutrinos [28], the Higgs [29], and gravity [30, 31].
The speed of light in particular has played a key role in both the conception of Lorentz symmetry and its early experimental tests. The continuing importance of electrodynamics to the subject is illustrated by the high precision with which the SME's photon sector is bounded. However, the most notable exception to these tight limits has been the SME $\tilde{\kappa}_{\text {tr }}$ coefficient, which parameterizes isotropic shifts in the speed of light. While the gap in precision to other laboratory constraints on electrodynamics was, until recently, at least 5 orders of magnitude, we recently published new results employing data from CERN's Large Electron Positron (LEP) collider and Fermilab's Tevatron, improving existing laboratory limits on $\tilde{\kappa}_{\text {tr }}$ by 3-4 orders of magnitude 32].

In this paper, we expand on our recent work to provide a detailed analysis of the sensitivity of collider experiments to $\tilde{\kappa}_{\mathrm{tr}}$. The outline of this paper is as follows. In Sec. [II, we review the basics of Lorentz-violating electrodynamics coupled to charged matter and present the basic idea behind the physics leading to our constraints on $\tilde{\kappa}_{\text {tr }}$. Section III employs the absence of vacuum Cherenkov radiation to limit positive values of $\tilde{\kappa}_{\text {tr }}$. Constraints on negative values of $\tilde{\kappa}_{\text {tr }}$, inferred from photon stability, are derived in Sec. IV] Section V]presents a brief summary and mentions future possibilities for measuring $\tilde{\kappa}_{\text {tr }}$, along with some of the associated projected bounds. Supplementary material is collected in two appendices. Unless noted otherwise, we work in natural units $c=\hbar=1$, and our convention for the metric signature is $(+,-,-,-)$.

\section{BASICS}

The photon and electron sectors of the minimal SME 11] are described by the Lagrangian

$$
\begin{aligned}
\mathcal{L}= & -\frac{1}{4} F^{2}-\frac{1}{4}\left(k_{F}\right)^{\kappa \lambda \mu \nu} F_{\kappa \lambda} F_{\mu \nu}+\left(k_{A F}\right)^{\mu} A^{\nu} \tilde{F}_{\mu \nu} \\
& +\frac{1}{2} i \bar{\psi} \Gamma^{\nu} \stackrel{\leftrightarrow}{D}_{\nu} \psi-\bar{\psi} M \psi
\end{aligned}
$$


where

$$
\begin{aligned}
\Gamma^{\nu} & \equiv \gamma^{\nu}+c_{e}^{\mu \nu} \gamma_{\mu}+d_{e}^{\mu \nu} \gamma_{5} \gamma_{\mu}, \\
M & \equiv m_{e}+b_{e}^{\mu} \gamma_{5} \gamma_{\mu}+\frac{1}{2} H_{e}^{\mu \nu} \sigma_{\mu \nu},
\end{aligned}
$$

$F^{\mu \nu}=\partial_{\mu} A_{\nu}-\partial_{\nu} A_{\mu}$ is the electromagnetic field-strength tensor, and $\tilde{F}^{\mu \nu}=(1 / 2) \epsilon^{\mu \nu \rho \sigma} F_{\rho \sigma}$ denotes its dual. The spinor $\psi$ describes electrons and positrons of mass $m_{e}$, and the usual $\mathrm{U}(1)$-covariant derivative is denoted by $D^{\mu}=\partial^{\mu}+i e A^{\mu}$. The SME coefficients $\left(k_{F}\right)^{\mu \nu \rho \lambda},\left(k_{A F}\right)^{\mu}$, $b_{e}^{\mu}, c_{e}^{\mu \nu}, d_{e}^{\mu \nu}$, and $H_{e}^{\mu \nu}$ control the extent of Lorentz and CPT violation.

In this work, we are primarily interested in the $\tilde{\kappa}_{\mathrm{tr}}$ component of $\left(k_{F}\right)^{\kappa \lambda \mu \nu}$, and will set $\left(k_{A F}\right)^{\mu}$ to zero. The $\left(k_{F}\right)^{\kappa \lambda \mu \nu}$ coefficient possesses the symmetries of the Riemann curvature tensor, and its double trace vanishes $\left(k_{F}\right)^{\kappa \lambda}{ }_{\kappa \lambda}=0$, leaving 19 independent components. To exhibit $\tilde{\kappa}_{\text {tr }}$, we decompose $\left(k_{F}\right)^{\kappa \lambda \mu \nu}$ such that the electromagnetic component of the Lagrangian (11) becomes:

$$
\begin{aligned}
\mathcal{L}= & \frac{1}{2}\left[\left(1+\tilde{\kappa}_{\mathrm{tr}}\right) \mathbf{E}^{2}-\left(1-\tilde{\kappa}_{\mathrm{tr}}\right) \mathbf{B}^{2}\right]+\frac{1}{2} \mathbf{E} \cdot\left(\tilde{\kappa}_{e+}+\tilde{\kappa}_{e-}\right) \cdot \mathbf{E} \\
& -\frac{1}{2} \mathbf{B} \cdot\left(\tilde{\kappa}_{e+}-\tilde{\kappa}_{e-}\right) \cdot \mathbf{B}+\mathbf{E} \cdot\left(\tilde{\kappa}_{o+}+\tilde{\kappa}_{o-}\right) \cdot \mathbf{B} .
\end{aligned}
$$

Here, the dimensionless parameter $\tilde{\kappa}_{\text {tr }}$ and the dimensionless and traceless $3 \times 3$ matrices $\tilde{\kappa}_{e-}, \tilde{\kappa}_{e+}, \tilde{\kappa}_{o+}$, and $\tilde{\kappa}_{o-}$ are defined in terms of the $\left(k_{F}\right)^{\kappa \lambda \mu \nu}$ coefficients [14] with $\tilde{\kappa}_{\text {tr }} \equiv(2 / 3)\left(k_{F}\right)_{\mu}{ }^{0 \mu 0}$. Note that the above decomposition of $\left(k_{F}\right)^{\kappa \lambda \mu \nu}$ into $\tilde{\kappa}$ coefficients is not manifestly coordinate independent: under changes of the observer inertial frame, the various $\tilde{\kappa}$ parameters mix. To facilitate comparisons between different experimental tests, a reference coordinate system must therefore be selected. A conventional choice is the Sun-centered celestial equatorial frame [14].

The parameterization determined by Eq. (3) is particularly intuitive because of its analogy to conventional electrodynamics in macroscopic media [11, 14]. For example, all $\tilde{\kappa}$ coefficients modify the photon dispersion relation, and thus the phase speed of light $c_{\mathrm{ph}}$. A subset of them, namely $\tilde{\kappa}_{o-}$ and $\tilde{\kappa}_{e+}$, affect each of the two electromagnetic-wave polarizations differently leading to birefringence. The absence of this type of birefringence in spectropolarimetric studies of cosmological sources constrains $\tilde{\kappa}_{o-}$ and $\tilde{\kappa}_{e+}$ at the level of $10^{-37}$ [33]. The remaining coefficients $\tilde{\kappa}_{e-}, \tilde{\kappa}_{o+}$, and $\tilde{\kappa}_{\text {tr }}$ arise from the

$$
\tilde{k}^{\mu \nu} \equiv\left(k_{F}\right)_{\alpha}^{\mu \alpha \nu}
$$

component. They lead to polarization-independent shifts in $c_{\mathrm{ph}}$, so that other types of measurements are necessary. A particularly sensitive measurement involves optical- or microwave-cavity experiments that search for parity-even anisotropies in $c_{\mathrm{ph}}$. Assuming negligible Lorentz violation effects on the cavity itself, these tests set limits on the elements of $\tilde{\kappa}_{e-}$ at the level of $10^{-17}$ [34]. The parity-odd matrix $\tilde{\kappa}_{o+}$ and the isotropic $\tilde{\kappa}_{\text {tr }}$ can be constrained indirectly with such measurements via higherorder effects: the laboratory is boosted with respect to the Sun-centered celestial equatorial frame, so that $\tilde{\kappa}_{e-}$, $\tilde{\kappa}_{o+}$, and $\tilde{\kappa}_{\text {tr }}$ mix. The absence of any observed resultant effect upon the cavity resonances yields $\tilde{\kappa}_{o+} \lesssim 10^{-13}$ [34], and $\left|\tilde{\kappa}_{\text {tr }}\right|<1.8 \times 10^{-8}[35]$. The latter had represented the best laboratory bound on $\tilde{\kappa}_{\text {tr }}$ prior to the recent paper [32] expanded here.

The present study exploits direct physical effects of $\tilde{\kappa}_{\text {tr }}$ to obtain improved constraints on this coefficient. The basic idea is that dispersion-relation modifications due to $\tilde{\kappa}_{\text {tr }}$ would not only change $c_{\mathrm{ph}}$, but also affect the kinematics of the electromagnetic vertex. In contrast to the conventional case, the three external legs of the vertex can go simultaneously on shell allowing various particle reactions to proceed that are normally forbidden by Lorentz symmetry. We focus on two such reactions, each occurring only for a specific sign of $\tilde{\kappa}_{\mathrm{tr}}$. The first is vacuum Cherenkov radiation

$$
f \rightarrow f+\gamma \text { for } \quad \tilde{\kappa}_{\text {tr }}>0,
$$

and the second is photon decay

$$
\gamma \rightarrow f+\bar{f} \quad \text { for } \quad \tilde{\kappa}_{\text {tr }}<0 .
$$

Here, $\gamma$ denotes a photon, $f$ a charged fermion, and $\bar{f}$ the corresponding antifermion. These processes are depicted in Fig. 1.

In what follows, we will explore how the observed absence of the reactions (5) and (6) provides bounds on $\tilde{\kappa}_{\text {tr }}$, expanding on our recently published paper on this subject 32]. Similar ideas have been exploited previously [16, 36], primarily in the context of purely kinematical dispersion-relation tests [37]. In the present case, the underlying SME Lagrangian permits the inclusion of dynamical features, such as the rate at which the reactions (5) and (6) proceed. Dynamical considerations are often necessary to obtain convincing and conservative results [38, 39].

In addition to $\tilde{\kappa}_{\text {tr }}$, other Lorentz-violating SME coefficients can lead to dispersion-relation modifications, and thus to the reactions (5) or (6). However, the effects of such additional coefficients can be safely neglected as long as their scale $\mathcal{S}$ is small compared to the $\tilde{\kappa}_{\text {tr }}$ constraint to be determined. The other relevant coefficients are those of the fermion $f$, the remaining $k_{F}$ components (i.e., the $\tilde{\kappa}$ matrices), and the $k_{A F}$ coefficient in Lagrangian (11). Since we will primarily consider $f$ to be an electron, the relevant matter-sector coefficients in the minimal SME are $b_{e}^{\mu}, c_{e}^{\mu \nu}, d_{e}^{\mu \nu}$, and $H_{e}^{\mu \nu}$ of the electron. With these considerations, the scale $\mathcal{S}$ is given by

$$
\mathcal{S} \equiv \max \left(\tilde{\kappa}_{e \pm}, \tilde{\kappa}_{o \pm}, \frac{k_{A F}}{m_{e}}, \frac{b_{e}}{m_{e}}, c_{e}, d_{e}, \frac{H_{e}}{m_{e}}\right) .
$$

Here, the absolute values of the individual components of the SME coefficients are implied, and $m_{e}$ the electron mass, as before.

To interpret and determine the scale $\mathcal{S}$ correctly, certain subtleties need to be taken into account. One 


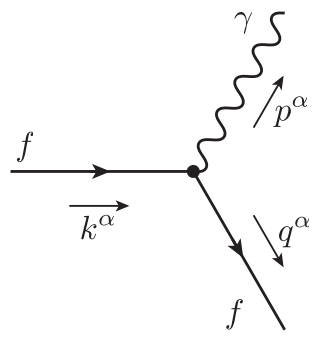

(a) Cherenkov effect

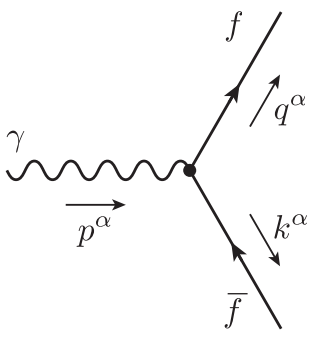

(b) photon decay
FIG. 1: Two possible electromagnetic-vertex configurations. Charged fermions and antifermions are denoted by $f$ and $\bar{f}$, respectively. The photon is labeled by $\gamma$. In the conventional case, energy-momentum conservation together with ordinary dispersion relations prohibits the three external legs from going simultaneously on-shell. In the presence of the Lorentzviolating $\tilde{\kappa}_{\mathrm{tr}}$ modification of the photon dispersion relation kinematically allows reactions (a) and (b) for $\tilde{\kappa}_{\text {tr }}>0$ and $\tilde{\kappa}_{\text {tr }}<0$, respectively.

such is that elements of the electron's $c_{e}^{\mu \nu}$ coefficient are physically equivalent to the nonbirefringent $\tilde{\kappa}$ parameters, as can be established by a coordinate redefinition. This means that only the anisotropic components of $c_{e}^{\mu \nu}$ should enter the determination of $\mathcal{S}$ because only anisotropic $\tilde{\kappa}$ 's occur in $\mathcal{S}$. More importantly, the equivalence of $c_{e}^{\mu \nu}$ and the nonbirefringent $\tilde{\kappa}$ 's implies that our ultimate constraint upon $\tilde{\kappa}_{\text {tr }}$ is, strictly speaking, a constraint upon the linear combination $\tilde{\kappa}_{\mathrm{tr}}-(4 / 3) c_{e}^{00}$. In what follows, we will often scale the coordinates such that $c_{e}^{00}=0$, but undo this special choice of scaling and reinstate $c_{e}^{00}$ when stating results. A more complete discussion of the above issues is contained in Appendix $\mathrm{A}$. The result of interest in the present context is that currently $\mathcal{S} \sim 10^{-13}$ dominated by the $\tilde{\kappa}_{o+}$ matrix coefficient 13 ]. So we may indeed focus on $\tilde{\kappa}_{\text {tr }}$ and ignore other types of Lorentz violation for photon-electron interactions.

We note in passing that vacuum Cherenkov radiation can also occur for antifermions and that further unconventional processes, such as fermion-antifermion annihilation into a single photon, are possible. Moreover, twophoton emission and absorption processes, synchrotron radiation, and inverse Compton processes can also be modified. Some of these effects have been employed in astrophysical contexts to extract general bounds on $\Delta c_{\mathrm{ph}} / c_{\mathrm{ph}}$ down to the $10^{-16}$ level [19].

\section{VACUUM CHERENKOV RADIATION}

In the present context, the vacuum Cherenkov effect (5) can only occur for positive $\tilde{\kappa}_{\text {tr }}$. To leading order, the modified dispersion relation for a photon with wave vector $p^{\mu} \equiv\left(E_{\gamma}, \vec{p}\right)$ is 14$]$

$$
E_{\gamma}^{2}-\left(1-\tilde{\kappa}_{\text {tr }}\right) \vec{p}^{2}=0
$$

We scale the coordinates such that the fermion dispersion relation remains conventional. Energy-momentum conservation for the process (5) then yields a threshold energy $E_{\mathrm{VCR}}$,

$$
E_{\mathrm{VCR}}=\frac{1-\tilde{\kappa}_{\mathrm{tr}}}{\sqrt{\left(2-\tilde{\kappa}_{\mathrm{tr}}\right) \tilde{\kappa}_{\mathrm{tr}}}} m=\frac{1}{\sqrt{2 \tilde{\kappa}_{\mathrm{tr}}}} m+\mathcal{O}\left(\sqrt{\tilde{\kappa}_{\mathrm{tr}}}\right)
$$

which corresponds to the kinetic energy of a fermion with mass $m$ moving as fast as photons obeying (8) in the vacuum [40]. For charges with energies above $E_{\mathrm{VCR}}$, vacuum Cherenkov radiation is kinematically allowed.

Here we constrain positive values of $\tilde{\kappa}_{\text {tr }}$ from the observed absence of vacuum Cherenkov radiation in nature. To set such constraints, vacuum Cherenkov radiation would have to be efficient enough to be observable. Close to the threshold energy $E_{\mathrm{VCR}}$, the dominant process is single-photon emission, such that the charge falls below threshold; an estimate for the corresponding rate is [40]:

$$
\Gamma_{\mathrm{VCR}}=\alpha Z^{2} m^{2} \frac{\left(E_{f}-E_{\mathrm{VCR}}\right)^{2}}{2 E_{f}^{3}},
$$

where $\alpha$ is the fine-structure constant, $Z$ the charge measured in multiples of the elementary charge, and $E_{f}$ the fermion energy. This shows the effect is undoubtedly efficient: for example, a $104.5 \mathrm{GeV}$ electron with an energy of $1 \%$ above the threshold (9) would reach subluminal speeds after traveling an average distance of $23 \mathrm{~cm}$. We therefore conclude that limits on $\tilde{\kappa}_{\text {tr }}$ can indeed be established from the observed absence at particle colliders of the vacuum Cherenkov effect for low-mass charges at the highest possible energies.

\section{A. Bounds from collider experiments}

Common to all analyses of collider experiments is a precise knowledge of the species and energy of the potential vacuum Cherenkov emitter. At present, the LEP experiment provides the best compromise between a charge's mass vs. its energy for terrestrial Cherenkov constraints. As a result, we can immediately determine that measurements of $E_{\mathrm{VCR}}$ derived from the LEP $e^{+} e^{-}$ beams will constrain $\tilde{k}^{\mu \nu}-2 c_{e}^{\mu \nu}$, independent of Lorentzviolating effects for other particles. As shown in Appendix A, for the energies attained at LEP $(\sim 100 \mathrm{GeV})$, $\tilde{\kappa}_{\text {tr }}-(4 / 3) c_{e}^{00}$ is the only SME coefficient combination that can contribute to vacuum Cherenkov radiation, permitting a significantly simplified study. An analysis of LEP data has the potential to yield rigorous one-sided improvements upon previous laboratory constraints on $\tilde{\kappa}_{\text {tr }}$.

The LEP collider was a circular particle accelerator approximately $27 \mathrm{~km}$ in circumference. This accelerator was an exquisitely precise and carefully controlled device with a relative uncertainty in the center-of-mass energy $\Delta E_{\mathrm{CM}} / E_{\mathrm{CM}}$ less than $2.0 \times 10^{-4}[41]$. To keep the 
uncertainty at this level, minute effects such as Earth tides, variations in the pressure of the local water table, and even seasonal variations in the volume of the nearby lake needed to be taken into account [41]. The highest laboratory-frame energy attained at LEP was $E_{\mathrm{LEP}}=104.5 \mathrm{GeV}$. We can obtain a first estimate for a limit on $\tilde{\kappa}_{\text {tr }}$ by arbitrarily setting $E_{\mathrm{VCR}}=100 \mathrm{GeV}$. Then, $104.5 \mathrm{GeV}$ electrons or positrons would fall below threshold after traveling approximately $1.2 \mathrm{~cm}$. This length is far shorter than the distance between superconducting radio-frequency cavities at LEP or even the dimensions $(5.8 \mathrm{~m})$ of each of the dipole bending magnets [41], so that such an effect would have been readily apparent. The observed absence of such effects at LEP implies $E_{\mathrm{VCR}}>100 \mathrm{GeV}$; together with Eq. (9), we then obtain $0 \leq \tilde{\kappa}_{\mathrm{tr}} \leq 1.3 \times 10^{-11}$. A more refined line of reasoning is presented in the next paragraphs.

At $E_{\mathrm{LEP}}=104.5 \mathrm{GeV}$, the energy loss due to conventional synchrotron radiation was $U_{0}=3.486 \mathrm{GeV}$ per electron or positron per turn [41]. The LEP circumference of $26659 \mathrm{~m}$ then predicts an average energy loss per distance travelled of

$$
\frac{d E_{\mathrm{syn}}}{d L}=2.580 \times 10^{-20} \mathrm{GeV}^{2} .
$$

One of the three energy-calibration methods at LEP relied upon the dependence of the synchrotron tune on the energy loss. For this reason, a precise determination of the energy loss was paramount. Deviations from the value (11) arise through parasitic-mode losses, finite beam size and other quadrupole effects, and losses in the corrector dipoles. The sum of these contributions is conservatively estimated to be $0.5 \mathrm{MeV}$ per turn per particle with at most a $20 \%$ uncertainty [41]. This implies that

$$
\frac{d E_{\mathrm{Cher}}}{d L} \leq 10^{-4} \frac{d E_{\mathrm{syn}}}{d L}
$$

where $d E_{\text {Cher }} / d L$ denotes the energy loss per distance due to vacuum Cherenkov radiation.

The final step is to determine a lower bound for $E_{\mathrm{VCR}}$ such that the inequality (12) together with the value (11) is satisfied. To this end, recall that for charges near $E_{\mathrm{VCR}}$ the dominant Cherenkov process for reaching subthreshold energies proceeds via single-photon emission (10). The energy loss per Cherenkov event must therefore be greater than $E-E_{\mathrm{VCR}}$. The average distance $L$ traversed by an electron before Cherenkov emission occurs is $1 / \Gamma_{\mathrm{VCR}}$. With Eq. (10), this yields

$$
\frac{d E_{\mathrm{Cher}}}{d L} \geq \alpha m_{e}^{2} \frac{\left(E_{\mathrm{LEP}}-E_{\mathrm{VCR}}\right)^{3}}{2 E_{\mathrm{LEP}}^{3}},
$$

where $m_{e}=5.11 \times 10^{-4} \mathrm{GeV}$ denotes the electron mass, as before. It follows that $E_{\mathrm{VCR}}$ can at most be $1.5 \mathrm{MeV}$ below $E_{\mathrm{LEP}}=104.5 \mathrm{GeV}$. With Eq. (91), we then obtain

$$
0 \leq \tilde{\kappa}_{\mathrm{tr}}-\frac{4}{3} c_{e}^{00} \leq 1.2 \times 10^{-11}
$$

explicitly including the contribution of $c_{e}^{00}$. The above reasoning also shows that the uncertainty in the bound (14) is primarily determined by the accuracy of the electron-energy measurement. As this limit is still much larger than the scale $\mathcal{S}$ defined in Eq. (7), other photon- or electron-sector coefficients are not further constrained by this reasoning. At the same time, this provides the justification for dropping these additional coefficients from our analysis.

\section{B. Cosmic-ray analyses}

Vacuum Cherenkov tests compare the respective group velocities $\vec{v}_{X}$ and $\vec{v}_{\gamma}$ of the charge $X$ and the photon $\gamma$. Since both particles may exhibit independent Lorentzviolating effects, vacuum Cherenkov radiation will typically depend on parameters originating from both the photon and the charge sectors of the SME. This means that any analysis of Lorentz-symmetry violation based on vacuum Cherenkov physics must address the following points: First, in the absence of independent constraints from other experiments, a Cherenkov analysis must incorporate all relevant Lorentz-symmetry violating coefficients from the photon as well as the charge. Second, the nature of the charge must be known, since it would otherwise be unclear as to which SME coefficients are actually constrained. The analysis of the previous subsection easily addresses the second point, as it involves the electrons and positrons at LEP. The first point is also addressed in detail in Appendix A, the availability of complementary experimental results leads to an estimation of the scale $\mathcal{S}$ in Eq. 7 which justifies dropping all but the single combination $\tilde{\kappa}_{\text {tr }}-(4 / 3) c_{e}^{00}$ from consideration.

Charged ultrahigh-energy cosmic rays (UHECRs) offer the potential to yield the tightest limits on positive values of $\tilde{\kappa}_{\text {tr }}$, as they possess energies orders of magnitude above those available in any laboratory [16]. Unfortunately, efforts to use observations of UHECRs to constrain Lorentz violation in the photon sector are currently beleaguered by a number of interpretational difficulties. Chief among them is the lack of certainty as to the composition of UHECR primaries, leading to an associated uncertainty as to which SME coefficients are constrained. Although the observed UHECR primaries are believed to be single protons, the possibility that the observations could be due to the scattering of more massive nuclei, highenergy photons, or Lorentz-violating particles exhibiting no or a qualitatively different Cherenkov effect, such as stable neutral pions or neutrons [36], cannot yet be excluded. This uncertainty will likely be ameliorated in coming years with continued observations.

If the UHECR primaries are found to be single protons, then in principle the analysis in Ref. [16] establishes that constraints on a combination of photon and proton SME coefficients at the $10^{-21} \ldots 10^{-22}$ level can be obtained. In contrast to our LEP-electron study, however, there is an insufficient number of complementary exper- 
imental bounds to solely focus on the nine parameters $2 c_{p}^{\mu \nu}-\tilde{k}^{\mu \nu}$ [42, 43]. For example, the $d_{p}^{\mu \nu}$ SME coefficient also affects the maximal attainable velocity of the proton and can therefore lead to vacuum Cherenkov radiation. But thus far, only two of its nine components have been bounded [22], so in general this coefficient needs to be taken into consideration when performing vacuum Cherenkov tests with protons.

If, instead, the UHECR primaries are identified as atomic nuclei such as $\mathrm{He},{ }^{12} \mathrm{C}$, or even ${ }^{56} \mathrm{Fe}$ nuclei, as is assumed in Ref. [16], various additional considerations are necessary. For example, the physical system under consideration now also contains neutrons in addition to photons and protons. This means that in principle neutron SME coefficients need to be considered as well. Moreover, the potential Cherenkov emitter is now a bound state without its own SME coefficients. One could introduce effective Lorentz-violating parameters for the nucleus, but this would hamper comparison with other limits on Lorentz violation for the photon, proton, or neutron. It is therefore preferable to determine the group velocity of the nucleus in terms of the proton's and neutron's SME coefficients via simplified nuclear modeling. To this end, it may be possible to employ the nuclear Schmidt model along the lines of the analysis in Ref. 222].

\section{PHOTON DECAY}

For negative $\tilde{\kappa}_{\text {tr }}$, the phase speed of light is greater than unity [44]. Vacuum Cherenkov radiation is then forbidden and cannot be used to set experimental limits. However, the kinematics of the electromagnetic vertex now allows photon decay into fermion-antifermion pairs (6). The dispersion relation (8) remains valid and establishes that photons with energies

$$
E_{\mathrm{pair}}=\frac{2 m}{\sqrt{\tilde{\kappa}_{\mathrm{tr}}\left(\tilde{\kappa}_{\mathrm{tr}}-2\right)}}=\sqrt{\frac{2}{-\tilde{\kappa}_{\mathrm{tr}}}} m+\mathcal{O}\left(\sqrt{\tilde{\kappa}_{\mathrm{tr}}}\right)
$$

or above are unstable, where $m$, as before, is the fermion mass. In Appendix B we derive the corresponding treelevel decay rate (B9), which to leading order in $\tilde{\kappa}_{\text {tr }}$ is

$$
\Gamma_{\text {pair }}=\frac{2}{3} \alpha E_{\gamma} \frac{m^{2}}{E_{\text {pair }}^{2}} \sqrt{1-\frac{E_{\text {pair }}^{2}}{E_{\gamma}^{2}}}\left(2+\frac{E_{\text {pair }}^{2}}{E_{\gamma}^{2}}\right) .
$$

Here, $E_{\gamma}$ denotes the photon energy and $\alpha$ is again the fine-structure constant [46]. The efficiency of this photon decay can be established by example: a $40 \mathrm{GeV}$ photon with energy $1 \%$ above threshold would decay after traveling an average distance of about $15 \mu \mathrm{m}$.

The above results show that we may obtain limits on negative values of $\tilde{\kappa}_{\text {tr }}$ from the existence of highenergy long-lived photons. As for the Cherenkov analysis, cosmic-ray observations provide the potential to reach the highest sensitivity. For example, primary photons from the Crab nebula with energies up to $80 \mathrm{TeV}$ have been reported by HEGRA [47]. Equation (15) then implies the possibility of one-sided limits on $\tilde{\kappa}$ coefficients at the $10^{-16}$ level. In addition to some of the nonbirefringent $\tilde{\kappa}$ matrices, certain SME coefficients of the electron cannot be neglected at these scales. In view of the small event sample for $\mathrm{TeV}$ gamma rays, the extraction of comprehensive and clean bounds on this potentially large number of SME coefficients appears unlikely at the present time. Estimates for limits from astrophysical $\mathrm{TeV}$ gamma rays would certainly be useful, but such an analysis lies outside the scope of the present study.

We focus instead on high-energy photons generated in Earth-based laboratories. As for vacuum Cherenkov radiation, the superior experimental control allows cleaner, more conservative limits albeit with reduced sensitivity. In this context, LEP becomes again one possible experiment to consider: e.g., past studies of quark-to-photon fragmentation involved a careful analysis of final-state photons in LEP scattering events. In such analyses, photons with energies up to $42 \mathrm{GeV}$ have been studied at OPAL [48], implying such photons survived long enough to interact normally with the OPAL calorimeter. Equation (15) then allows limits at the level $-3 \times 10^{-10} \lesssim \tilde{\kappa}_{\text {tr }}$. Other LEP studies at L3 [49] and OPAL [50] detectors, which were optimized for QED precision tests, have measured pair annihilation $e^{+} e^{-} \rightarrow \gamma \gamma$ at center-of-mass energies up to $209 \mathrm{GeV}$. This would yield an even better bound of $-5 \times 10^{-11} \lesssim \tilde{\kappa}_{\mathrm{tr}}$.

Nevertheless, the highest energies at terrestrial accelerators are not reached with electrons but with hadrons. For example, Fermilab's Tevatron $p \bar{p}$ collider produces center-of-mass energies up to $1.96 \mathrm{TeV}$ and offers therefore excellent potential for producing high-energy photons. One particular process, namely isolated-photon production with an associated jet, is of importance for QCD studies and has therefore been investigated with the D0 detector. In this context, photons of energies up to $442 \mathrm{GeV}$ have been observed [51]. The implied stability of photons with such energies suggests an estimate of $-3 \times 10^{-12} \lesssim \tilde{\kappa}_{\mathrm{tr}}$. However, the small number of events observed at this energy did not warrant inclusion into these QCD investigations.

Our present analysis uses only D0 photon data at lower energies, where comparisons to QCD predictions were made. With this conservative restriction, photonenergy bins up to $340.5 \mathrm{GeV}$ were measured [52]. For this data, the aforementioned jet-plus-photon production was measured as a function of $E_{\gamma}$ in four angular regions. These four directional configurations were characterized by the photon and jet pseudorapidities $y^{\gamma}$ and $y^{\text {jet }}$. The largest deviations between experiment and QCD theory in the $340.5 \mathrm{GeV}$ energy bin occurred in the $\left\{\left|y^{\text {jet }}\right|<0.8, y^{\gamma} y^{\text {jet }}<0\right\}$ angular region [52]. The measured cross section was about $52 \%$ of the QCD prediction. The relative uncertainties in the experimental value were $46.1 \%$ statistical, $12.9 \%$ systematic, and a $7.8 \%$ normalization error [52]. To account for uncertainties, the employed theoretical scales were varied by a factor of 2 , 
which led to a relative spread of about $11 \%$ for theoretical predictions [52]. Combining these errors in quadrature yields an overall relative uncertainty of about $50 \%$. The experiment-to-theory ratio in the $340.5 \mathrm{GeV}$ energy bin is therefore $0.52 \pm 0.26$ for the selected angular configuration. We can thus estimate that at least $26 \%$ of the produced photons have reached the detector.

The layout of the D0 detector implies that measured photons traverse a minimum distance of $l_{\min } \simeq 78 \mathrm{~cm}$ : they have to travel through various drift chambers and the transition-radiation detector before they interact and are detected in the central calorimeter [53]. With the above photon-flux estimate, we then obtain

$$
\exp \left(-\Gamma_{\text {pair }} l_{\text {min }}\right) \geq 0.26 .
$$

The $340.5 \mathrm{GeV}$ energy bin extended from $300 \mathrm{GeV}$ to $400 \mathrm{GeV}$. We therefore conservatively take $E_{\gamma}=300$ $\mathrm{GeV}$ in our analysis. With Eq. (16), we then find that $E_{\text {pair }}$ cannot be more than about $0.1 \mathrm{keV}$ below $E_{\gamma}$. Explicitly including the contribution of $c_{e}^{00}$, we therefore conclude that

$$
-5.8 \times 10^{-12} \leq \tilde{\kappa}_{\mathrm{tr}}-\frac{4}{3} c_{e}^{00} .
$$

The uncertainty in the constraint (18) is essentially determined by the accuracy of the photon-energy measurement. As with the Cherenkov bound, the limit (18) is larger than the scale $\mathcal{S}$, so other photon- or electronsector coefficients are not further constrained by this argument. At the same time, this justifies the exclusion of these additional coefficients from our study.

\section{SUMMARY AND OUTLOOK}

In this paper, we have considered new physical effects arising from a Lorentz-violating CPT-even deviation of the phase speed of light $c_{\mathrm{ph}}$ from its conventional value c. At the theoretical level, such a deviation is controlled by the $\tilde{\kappa}_{\text {tr }}$ coefficient of the SME. This coefficient is defined with respect to the Sun-centered celestial equatorial coordinate system, in which the phase-speed deviation is isotropic. At the phenomenological level, a positive value for $\tilde{\kappa}_{\text {tr }}$ would lead to vacuum Cherenkov radiation (5) at the rate (10) for charges with energies above the threshold (9); whereas a negative value would cause photondecay (6) at the rate (16) for photons with energies above the threshold (15).

We have exploited the fact that both phenomena are efficient threshold effects to extract constraints on $\tilde{\kappa}_{\text {tr }}$ from the nonobservation of vacuum Cherenkov radiation and photon decay. In particular, the absence of the Cherenkov effect at LEP leads to the bound (14), and from the stability of photons at the Tevatron the constraint (18) can be inferred. These results give the combined conservative limit

$$
-5.8 \times 10^{-12} \leq \tilde{\kappa}_{\mathrm{tr}}-\frac{4}{3} c_{e}^{00} \leq 1.2 \times 10^{-11} .
$$

This limit represents an improvement of previous laboratory bounds by $3-4$ order of magnitude.

There are various ways for complementary or improved bounds to be set on $\tilde{\kappa}_{\text {tr }}$. For instance, planned low-energy laboratory tests could reach a level of $10^{-11}$ or better [17]. Another idea is to exploit photon triple splitting, as it is known that the amplitude for this effect is nonzero in the presence of $c^{\mu \nu}$ Lorentz violation [54]. This effect does not involve a threshold, and so high energies are not necessarily required.

Other future terrestrial bounds could employ the absence of vacuum Cherenkov radiation and photon decay at even higher energies than the ones considered here. One example would be the prospective International Linear Collider. If we take the laboratory-frame energy to be $500 \mathrm{GeV}$, the International Linear Collider gives a projected one-sided Cherenkov limit of $0 \leq \tilde{\kappa}_{\mathrm{tr}}-(4 / 3) c_{e}^{00} \leq$ $5.2 \times 10^{-13}$. Similarly, the Large Hadron Collider will reach about 7 times the energy of the Tevatron. Under the assumption that the energy of produced photons scales by the same factor, the limit (18) can be tightened by a factor of 50 . Other improvements of the photondecay bound would be possible with a dedicated D0 (or possibly Large Hadron Collider) analysis: Ultrahighenergy events not considered for QCD tests could be used because the statistics of such events is not of primary importance for photon-decay studies. Moreover, the end of the photon-energy spectrum could be exploited more efficiently by avoiding large energy bins.

We note that during the preparation of this manuscript, Brett Altschul performed a more detailed analysis of synchrotron radiation processes at LEP, obtaining an improved two-sided limit on isotropic violations of Lorentz symmetry for light relative to electrons of $\left|\tilde{\kappa}_{\mathrm{tr}}-(4 / 3) c_{e}^{00}\right| \leq 1.2 \times 10^{-15}[55]$.

The largest potential for improved bounds on $\tilde{\kappa}$ coefficients - in the context of both vacuum Cherenkov radiation and photon decay - lies probably in UHECR physics [16, 46] : e.g., with a more reliable identification of the UHECR primary particle, observations at still higher energies, better coverage of the sky with more events, and data analysis allowing for Lorentz violation in both the primary and the decay products should open an avenue to tap this potential more completely. A fundamental limit on the experimental reach is that the Universe becomes opaque to cosmic rays above certain thresholds due to processes such as Greisen-Zatsepin-Kuzmin suppression or scattering from IR photons.

\section{Acknowledgments}

R.L. is grateful to B. Altschul, F.R. Klinkhamer, and M. Schreck for helpful discussions. This work is supported in part by the European Commission under Grant No. MOIF-CT-2005-008687, by CONACyT under Grant No. 55310, and by the National Science Foundation. 


\section{APPENDIX A: COORDINATE RESCALINGS}

In this appendix, we present the details of our determination of the scale $\mathcal{S}$ defined in Eq. (7) at which Lorentz violation has been constrained for photons relative to electrons, based upon limits obtained from terrestrial tests. To accomplish this, we compare the results of experiments reporting bounds on Lorentz violation for photons, electrons, and protons. Making use of these bounds is somewhat complicated by the assumptions made regarding the possibility of Lorentz violation in other sectors of the SME.

Measurements of shifts and anisotropies in the vacuum speed of light must be defined in terms of the velocity of a chosen reference particle. In the SME, the limiting velocity of any such reference particle is also subject to Lorentz-violating shifts and anisotropies. As a result, constraints upon the deviation of the speed of light based upon interactions of light with electrons must be narrowly interpreted as limits on the difference between the degree to which Lorentz symmetry is violated in each sector. This is a general feature of all tests of Lorentz symmetry, which generally must be described as setting limits on combinations of coefficients associated with not one, but all involved particle species. In this sense, the number of independent SME coefficients controlling the type and extent of Lorentz violation in a given physical system is increased.

Under certain circumstances, various SME coefficients describing a given system may be physically equivalent and can therefore not be distinguished. From a mathematical viewpoint, there typically exist canonical transformations that can eliminate one coefficient in favor of the other. From an experimental viewpoint, this means that such coefficients cannot be bounded or measured independently in the physical system in question. In such a case, the number of independent SME coefficients is therefore effectively reduced.

It is the interplay of the above two issues that is often unappreciated in the literature. The interpretation of experimental constraints therefore requires special care. In the present context, the former issue has been discussed in various places in main text. The latter issue is not only paramount for the precise formulation of our actual bounds, but also for the interpretation of existing constraints necessary for the determination of the scale $\mathcal{S}$. The particular issue to be clarified in this appendix concerns the $\tilde{k}^{\mu \nu}$ coefficient (i.e., $\tilde{\kappa}_{e-}, \tilde{\kappa}_{o+}, \tilde{\kappa}_{\text {tr }}$ ) and $c^{\mu \nu}$-type coefficients. To simplify the discussion, we will set all other types of SME coefficients to zero in what follows.

In the context of the SME for one-flavor QED, a theory characterized by a nonzero symmetric, traceless $c_{e}^{\mu \nu}$ tensor and a vanishing $\left(k_{F}\right)^{\kappa \lambda \mu \nu}$ tensor exhibits the same phenomenology as a theory in which $c_{e}^{\mu \nu}=0[14,56]$ and

$$
\left(k_{F}\right)^{\mu \nu \rho \sigma}=\frac{1}{2}\left(\eta^{\mu \rho} \tilde{k}^{\nu \sigma}-\eta^{\mu \sigma} \tilde{k}^{\nu \rho}+\eta^{\nu \sigma} \tilde{k}^{\mu \rho}-\eta^{\nu \rho} \tilde{k}^{\mu \sigma}\right),
$$

provided that

$$
\tilde{k}^{\mu \nu}=-2 c_{e}^{\mu \nu}
$$

at linear order. Thus, a $\tilde{k}^{\mu \nu}$ model is physically equivalent to a $c_{e}^{\mu \nu}$ model if the models are related by Eq. (A2). This fact can also be formally established via coordinate rescalings [14, 56]. We may use this freedom to select a particularly convenient scaling of the coordinates to simplify calculations. For example, our analysis in the main text is performed within a $\tilde{k}^{\mu \nu}$ model (i.e., the coordinates are scaled such that $c_{e}^{\mu \nu}=0$ ), whereas our photondecay calculation in Appendix B employs a $c_{e}^{\mu \nu}$ model (i.e., the coordinates are rescaled such that $\tilde{k}^{\mu \nu}=0$ ). One way of quoting results, such as experimental constraints, is to employ a particular coordinate scaling and clearly state this special scaling choice together with the actual result.

The choices of rescaling form a continuous set and are not only confined to the two canonical cases of $c_{e}^{\mu \nu}=0$ and $\tilde{k}^{\mu \nu}=0$ discussed above. An infinite number of coordinate scalings with both $c_{e}^{\mu \nu} \neq 0$ and $\tilde{k}^{\mu \nu} \neq 0$ can certainly be selected. In the present context, one can show that with such general rescalings, physical effects can only depend upon (and thus provide bounds for) the value of $2 c_{e}^{\mu \nu}-\tilde{k}^{\mu \nu}$. This is intuitively reasonable because the two Lorentz-violating effects considered in this work, vacuum Cherenkov radiation and photon decay, depend only on certain velocity differences between the electron and the photon. Note in particular that the combination $2 c_{e}^{\mu \nu}-\tilde{k}^{\mu \nu}$ does not pertain to a particular choice of scale for the coordinates and therefore provides a second way to quote results that is coordinate-scaling independent. When formulating our final bounds in the main text, we have adopted this latter choice of stating results.

Other experimental tests of Lorentz and CPT symmetry are not generally confined to one-flavor QED; other particle species are often involved. In such situations, the above analysis is readily generalized: we may choose one particle species to serve as the reference "ruler," and thus work in a coordinate system in which that species' $c^{\mu \nu}$ coefficient (or $\tilde{k}^{\mu \nu}$, if the reference is light) is zero.

With these considerations, we estimate the value $\mathcal{S}$ provided by terrestrial experiments for the interaction of light with electrons. Specifically, we will use the results of a Cs-fountain clock experiment [23], and those of a series of tests involving optical resonators [34]. These tests are sensitive to Lorentz violation in conventional matter, which is made up of protons, neutrons, and electrons, interacting electromagnetically. In this four-species systems, the Lorentz-violating effects under consideration are described by four sets of SME coefficients: $c_{p}^{\mu \nu}, c_{n}^{\mu \nu}$, $c_{e}^{\mu \nu}$, and $\tilde{k}^{\mu \nu}$, where the subscripts $p, n$, and $e$ respectively denote the coefficients of protons, neutrons, and electrons. One of these four sets of terms may be eliminated by a scaling of coordinates, and so in practice only three of these sets of parameters may independently contribute to the physics. 
Although the Cs-fountain test [23] involves protons, neutrons, electrons, and electromagnetism, the observed frequencies turn out to be only sensitive to the value of $2 c_{p}^{\mu \nu}-\tilde{k}^{\mu \nu}$. The constraints upon the eight spatially anisotropic components are at the level of

$$
\left|2 c_{p}^{\mu \nu}-\tilde{k}^{\mu \nu}\right|<10^{-21} \ldots 10^{-25} .
$$

We note that these results are presented in Ref. 23] in coordinates such that $\tilde{k}^{\mu \nu}=0$, which corresponds to using light as a reference. In coordinates with protons as the reference (i.e., $c_{p}^{\mu \nu}=0$ ), the Cs-fountain experiment provides the constraint $\left|\tilde{k}^{\mu \nu}\right|<10^{-21} \ldots 10^{-25}$ for the anisotropic components of $\tilde{k}^{\mu \nu}$.

Next, we consider optical-resonator experiments [34], which measure the resonance frequencies $\nu$ of light propagating in vacuum inside two orthogonally oriented FabryPérot cavities. As previously shown [13, 15], these experiments are sensitive to spatial anisotropies in the speed of light $\left(\tilde{k}^{\mu \nu}\right)$ and to variations in the dimensions of the resonators themselves. The cavity size is primarily determined by the electromagnetic interactions in the chemical bonds. It therefore follows that the neutron's contribution to the cavity size must be suppressed because it is uncharged, having only a magnetic moment. Moreover, the cavities are made of fused silica $\mathrm{SiO}_{2}$, and the common isotopes of oxygen and silicon have even numbers of neutrons and spin zero. Pairing effects would therefore tend to further suppress the influence of the neutron spin, and so we conclude that the cavity frequencies should be largely unaffected by $c_{n}^{\mu \nu}$.

Bearing this suppression of neutron effects in mind, two independent combinations of parameters remain that can influence the observable $F$ determined from the cavity frequencies $\nu$. This observable must therefore be given, to leading order, by an expression of the form

$$
F=\text { const. }+A_{\mu \nu}\left(2 c_{e}^{\mu \nu}-\tilde{k}^{\mu \nu}\right)+B_{\mu \nu}\left(2 c_{p}^{\mu \nu}-\tilde{k}^{\mu \nu}\right),
$$

where $A_{\mu \nu}$ and $B_{\mu \nu}$ are constants. Constraints on the anisotropic pieces in Eq. (A4) at the $10^{-13} \ldots 10^{-17}$ level can be obtained by these cavity tests 34 .

The $B_{\mu \nu}$ term in Eq. (A4) can be dropped from these optical-resonator bounds for the following reason: The constants $A_{\mu \nu}$ and $B_{\mu \nu}$ are likely to be of similar size, as there appears to be no convincing argument suggesting that the SME effects in one of the three involved particle species would dominate the length of chemical bonds. For certain sample chemical bonds including fused silica, this has indeed been verified [57]. The next step is to observe that the independent Cs-fountain bound (A3) places a much tighter constrained on the coefficient combination multiplying $B_{\mu \nu}$ than the best sensitivity $10^{-17}$ of the optical-resonator test. We thus conclude that

$$
\left|2 c_{e}^{\mu \nu}-\tilde{k}^{\mu \nu}\right|<10^{-13} \ldots 10^{-17}
$$

follows from the cavity experiments [34]. It is understood that this bound refers to the anisotropic components of
$2 c_{e}^{\mu \nu}-\tilde{k}^{\mu \nu}$. We remark that Ref. [34] chooses to state the resulting experimental limit assuming scaled coordinates such that $c_{e}^{\mu \nu}=0$.

The constraint (A5) taken together with Refs. [13, 20] establish that $\mathcal{S} \sim 10^{-13}$, dominated by the contribution of the parity-odd $\tilde{\kappa}_{o+}$ and $c_{e}^{0 J}$ coefficients. Because the limit we will derive on isotropic $\tilde{\kappa}_{\text {tr }}$ component lies above this scale, we may indeed drop all other Lorentz-violating corrections from our analysis.

\section{APPENDIX B: PHOTON-DECAY RATE}

Photon-decay rates in the presence of Lorentz violation have been determined [58] for the dimension-three ChernSimons type SME coefficient $\left(k_{A F}\right)^{\mu}$ which governs photon triple splitting. In this appendix, we derive the treelevel photon-decay rate into a fermion-antifermion pair arising from the dimension-four SME $\tilde{\kappa}_{\text {tr }}$ coefficient, appropriate for our purposes.

The starting point is a model with Lorentz-violating photons and conventional charged leptons. In the present situation it is convenient to consider a physically equivalent model constructed with the coordinate redefinition discussed in Sec. II and Appendix A. In particular, we remove all Lorentz violation from the photon sector at the cost of introducing a Lorentz-breaking $c_{e}^{\mu \nu}$ coefficient in the lepton sector:

$$
\mathcal{L}^{\prime}=\frac{1}{2} i \bar{\psi}\left(\gamma^{\mu}+c_{e}^{\mu \nu} \gamma_{\nu}\right) \stackrel{\leftrightarrow}{D_{\mu}} \psi-m \bar{\psi} \psi-\frac{1}{4} F^{2}
$$

where $D_{\mu}=\partial_{\mu}+i e A_{\mu}$ is the usual covariant derivative. The Lorentz-violating SME coefficient $c_{e}^{\mu \nu}$ is given in explicit form as

$$
c_{e}^{\mu \nu}=-\frac{1}{4} \tilde{\kappa}_{\mathrm{tr}} \operatorname{diag}(3,1,1,1) .
$$

The advantage of the above description (B1) for $\tilde{\kappa}_{\mathrm{tr}}$ Lorentz violation is the following. Perturbation theory in quantum field theory relies on the quantization of the free-field sectors of the model. For Lorentz-violating photons, such a quantization is lacking, but the quantization of SME fermions is comparatively well understood [11]. Moreover, we may employ the methodology and notation of a previous tree-level calculation involving $c_{e}^{\mu \nu}$ fermions [59].

Since $\tilde{\kappa}_{\mathrm{tr}}-(4 / 3) c_{e}^{00}$ is nonzero, the lepton sector of the model (B1) in our chosen coordinates contains unconventional time derivatives. The time evolution of $\psi$ can then be nonunitary, so its asymptotic states cannot directly be identified with physical free-particle states. A standard approach to avoid this potential interpretational difficulty is a redefinition of the spinor field chosen to eliminate the additional time derivatives [59, 60]. In the present situation, the field redefinition amounts to a rescaling

$$
\psi \equiv \frac{1}{\sqrt{1-\frac{3}{4} \tilde{\kappa}_{\mathrm{tr}}}} \chi
$$




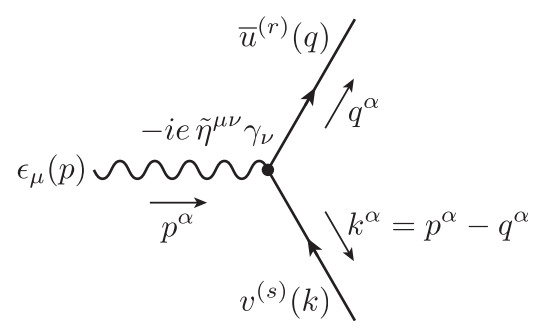

FIG. 2: Tree-level Feynman diagram for photon decay. Lorentz-violating effects are contained in the modified dispersion relation for the lepton and antilepton 4-momenta $q^{\alpha}$ and $k^{\alpha}$, respectively, as well as in the electromagnetic vertex containing $\tilde{\eta}_{\mu \nu}$.

so it is not strictly mandatory. We nevertheless implement the redefinition (B3) for compatibility with previous studies [59] of this type. The Lagrangian (B1) then becomes

$$
\mathcal{L}=\frac{1}{2} i \bar{\chi} \tilde{\eta}^{\mu \nu} \gamma_{\nu} \stackrel{\leftrightarrow}{D_{\mu}} \chi-\tilde{m} \bar{\chi} \chi-\frac{1}{4} F^{2},
$$

where we have defined

$$
\begin{aligned}
\tilde{m} & \equiv \frac{m}{1-\frac{3}{4} \tilde{\kappa}_{\mathrm{tr}}}, \\
\tilde{\eta}^{\mu \nu} & \equiv \operatorname{diag}(1,-\lambda,-\lambda,-\lambda), \\
\lambda & \equiv \frac{1+\frac{1}{4} \tilde{\kappa}_{\mathrm{tr}}}{1-\frac{3}{4} \tilde{\kappa}_{\mathrm{tr}}} .
\end{aligned}
$$

The field redefinition (B3) is a canonical transformation, and as such it leaves unchanged the physics. In particular, the free fermions in model (B1) possess the same dispersion relation as those in (B4).

Because $\tilde{\kappa}_{\text {tr }}$ is frame dependent, Eq. (B3) is not manifestly Lorentz coordinate covariant. Therefore, the specific form of Lagrangian (B4) holds only in the frame in which the field redefinition has been performed 61]. Note that the Lagrangians (B1) and (B4) are singular for $\tilde{\kappa}_{\mathrm{tr}}=-4$ and $\tilde{\kappa}_{\mathrm{tr}}=4 / 3$ while the dispersion relation (8) is singular at $\tilde{\kappa}_{\mathrm{tr}}=1$. This difference arises because the coordinate rescaling used to generate Lagrangian (B1) from the original $\tilde{\kappa}_{\text {tr }}$ model has been implemented only at leading order in $\tilde{\kappa}_{\mathrm{tr}}$.

The Feynman rules can now be inferred from the Lagrangian (B1). The appropriate tree-level Feynman di- agram for photon decay is depicted in Fig. 2. For the corresponding matrix element, we obtain

$$
i \mathcal{M}_{r s}=-i e \epsilon_{\mu}(p) \tilde{\eta}^{\mu \nu} \bar{u}^{(r)}(q) \gamma_{\nu} v^{(s)}(k)
$$

where the various polarization and momentum assignments are defined in Fig. 22 The next step is the calculation of $\left|\mathcal{M}_{r s}\right|^{2}$ followed by the usual summation over final spin states and averaging over the initial photon polarizations $\overline{|\mathcal{M}|^{2}} \equiv(1 / 2) \sum_{\epsilon} \sum_{r, s}\left|\mathcal{M}_{r s}\right|^{2}$. We obtain

$\overline{|\mathcal{M}|^{2}}=e^{2}\left[4 \tilde{m}^{2}+2 \lambda^{2}\left(1-\lambda^{2}\right)\left(\vec{q}^{2}+\vec{k}^{2}\right)+\left(1-\lambda^{2}\right)^{2} E_{\gamma}^{2}\right]$,

where $E_{\gamma}=|\vec{p}|$ is the photon energy and $\vec{q}$ and $\vec{k}$ are the lepton and antilepton 3-momenta, respectively. To arrive at this result, energy-momentum conservation, the usual relation for photon-polarization sums, and trace identities for Dirac matrices have been used. Moreover, we have employed the results for SME spinor projectors in Ref. [59] with the normalization chosen such that $N(\vec{q})=2 E_{q}=2 \sqrt{\tilde{m}^{2}+\lambda^{2} \vec{q}^{2}}$, etc.

The final step is the phase-space integration. In the conventional Lorentz-symmetric case, the decay rate for massive particles is defined in the particle's rest frame with a kinematic factor inversely proportional to its mass. This procedure cannot be applied to present massless case. We adopt instead the convention [54] to define the decay rate in terms of the photon energy $E_{\gamma}$ in the Suncentered celestial equatorial frame:

$$
\Gamma_{\text {pair }}=\frac{1}{4 \pi^{2}} \frac{1}{2 E_{\gamma}} \int \frac{d^{3} q}{2 E_{q}} \frac{d^{3} k}{2 E_{k}} \overline{|\mathcal{M}|^{2}} \delta^{(4)}(p-q-k) .
$$

This yields

$\Gamma_{\text {pair }}=\alpha \frac{\left[\tilde{\kappa}_{\mathrm{tr}}\left(\tilde{\kappa}_{\mathrm{tr}}-4\right) E_{\gamma}^{2}+4 m^{2}\right] \sqrt{\tilde{\kappa}_{\mathrm{tr}}\left(\tilde{\kappa}_{\mathrm{tr}}-4\right) E_{\gamma}^{2}-8 m^{2}}}{\frac{3}{8} E_{\gamma}^{2}\left(4+\tilde{\kappa}_{\mathrm{tr}}\right)\left(4-3 \tilde{\kappa}_{\mathrm{tr}}\right) \sqrt{\tilde{\kappa}_{\mathrm{tr}}\left(\tilde{\kappa}_{\mathrm{tr}}-4\right)}}$

for the exact tree-level decay rate within the context of Lagrangian (B4). Equation (B9) applies only for perturbative $\tilde{\kappa}_{\text {tr }}<0$ and for photons above threshold. We remark that undoing our initial coordinate redefinition would generate subleading corrections to Eq. (B9).
[1] For recent reviews of various experimental and theoretical approaches to Lorentz violation see, e.g., CPT and Lorentz Symmetry, edited by V.A. Kostelecký (World Scientific, Singapore, 1999); CPT and Lorentz Symmetry II, edited by V.A. Kostelecký (World Scientific, Singapore, 2002); CPT and Lorentz Symmetry III, edited by V.A. Kostelecký (World Scientific, Singapore, 2005); CPT and Lorentz Symmetry IV, edited by V.A. Kost- elecký (World Scientific, Singapore, 2008); R. Bluhm, Lect. Notes Phys. 702, 191 (2006) hep-ph/0506054; D.M. Mattingly, Living Rev. Rel. 8, 5 (2005).

[2] V.A. Kostelecký and S. Samuel, Phys. Rev. D 39, 683 (1989); V.A. Kostelecký and R. Potting, Nucl. Phys. B 359, 545 (1991).

[3] See, e.g., I. Mocioiu, M. Pospelov, and R. Roiban, Phys. Lett. B 489, 390 (2000); S.M. Carroll, J.A. Harvey, V.A. 
Kostelecký, C.D. Lane, and T. Okamoto, Phys. Rev. Lett. 87, 141601 (2001); C.E. Carlson, C.D. Carone, and R.F. Lebed, Phys. Lett. B 518, 201 (2001); A. Anisimov, T. Banks, M. Dine, and M. Graesser, Phys. Rev. D 65, 085032 (2002).

[4] O. Bertolami, R. Lehnert, R. Potting, and A. Ribeiro, Phys. Rev. D 69, 083513 (2004); V.A. Kostelecký, R. Lehnert, and M.J. Perry, Phys. Rev. D 68, 123511 (2003); R. Jackiw and S.-Y. Pi, Phys. Rev. D 68, 104012 (2003).

[5] See, e.g, J. Alfaro, H.A. Morales-Técotl, and L.F. Urrutia, Phys. Rev. Lett. 84, 2318 (2000); F.R. Klinkhamer and C. Rupp, Phys. Rev. D 70, 045020 (2004); G. Amelino-Camelia et al., AIP Conf. Proc. 758, 30 (2005); N.E. Mavromatos, Lect. Notes Phys. 669, 245 (2005).

[6] C.D. Froggatt and H.B. Nielsen, hep-ph/0211106.

[7] J.D. Bjorken, Phys. Rev. D 67, 043508 (2003).

[8] See, e.g., C.P. Burgess et al., JHEP 0203, 043 (2002); A.R. Frey, JHEP 0304, 012 (2003); J. Cline and L. Valcárcel, JHEP 0403, 032 (2004).

[9] See, e.g., V.A. Kostelecký and S. Samuel, Phys. Rev. D 42, 1289 (1990); N. Arkani-Hamed et al., JHEP 0405, 074 (2004); M.V. Libanov and V.A. Rubakov, JHEP 0508, 001 (2005); G. Dvali, O. Pujolas, and M. Redi, Phys. Rev. D 76, 044028 (2007); S. Dubovsky, P. Tinyakov, and M. Zaldarriaga, JHEP 0711, 083 (2007).

[10] V.A. Kostelecký and R. Potting, Phys. Rev. D 51, 3923 (1995).

[11] D. Colladay and V.A. Kostelecký, Phys. Rev. D 55, 6760 (1997); Phys. Rev. D 58, 116002 (1998); V.A. Kostelecký, Phys. Rev. D 69, 105009 (2004).

[12] R. Bluhm and V.A. Kostelecký, Phys. Rev. D 71, 065008 (2005); R. Bluhm, S.H. Fung, and V.A. Kostelecký, Phys. Rev. D 77, 065020 (2008); V.A. Kostelecký and R. Potting, Gen. Rel. Grav. 37, 1675 (2005).

[13] V.A. Kostelecký and N. Russell, arXiv:0801.0287.

[14] V.A. Kostelecký and M. Mewes, Phys. Rev. Lett. 87, 251304 (2001); Phys. Rev. D 66, 056005 (2002).

[15] J.A. Lipa, J.A. Nissen, S. Wang, D.A. Stricker, and D. Avaloff, Phys. Rev. Lett. 90, 060403 (2003); H. Müller, S. Herrmann, C. Braxmaier, S. Schiller, and A. Peters, Phys. Rev. Lett. 91, 020401 (2003); P. Wolf et al., Gen. Rel. Grav. 36, 2351 (2004); Phys. Rev. D 70, 051902(R) (2004); M.E. Tobar, P. Wolf, A. Fowler, and J.G. Hartnett, Phys. Rev. D 71, 025004 (2005); S. Herrmann, A. Senger, E. Kovalchuk, H. Müller, and A. Peters, Phys. Rev. Lett. 95, 150401 (2005); P.L. Stanwix, M.E. Tobar, P. Wolf, C.R. Locke, and E.N. Ivanov, Phys. Rev. D 74, 081101(R) (2006); H. Müller et al., Phys. Rev. Lett. 99, 050401 (2007); S. Reinhardt et al., Nature Physics 3, 861 (2007); S.M. Carroll, G.B. Field, and R. Jackiw, Phys. Rev. D 41, 1231 (1990); R. Jackiw and V.A. Kostelecký, Phys. Rev. Lett. 82, 3572 (1999); V.A. Kostelecký and M. Mewes, Phys. Rev. Lett. 97, 140401 (2006); Phys. Rev. Lett. 99, 011601 (2007); Q.G. Bailey and V.A. Kostelecký, Phys. Rev. D 70, 076006 (2004); C.D. Carone, M. Sher, and M. Vanderhaeghen, Phys. Rev. D 74, 077901 (2006); B. Altschul, Phys. Rev. Lett. 96, 201101 (2006); Phys. Rev. Lett. 98, 041603 (2007); Phys. Rev. D 75, 105003 (2007).

[16] F.R. Klinkhamer and M. Risse, Phys. Rev. D 77, 016002 (2008); Phys. Rev. D 77, 117901 (2008).

[17] M.E. Tobar, P. Wolf, A. Fowler, and J.G. Hartnett, Phys. Rev. D 71, 025004 (2005) [Erratum-ibid. D 75, 049902 (2007)].
[18] H. Dehmelt, R. Mittleman, R.S. Van Dyck, and P. Schwinberg, Phys. Rev. Lett. 83, 4694 (1999); R.K. Mittleman, I.I. Ioannou, H.G. Dehmelt, and N. Russell, Phys. Rev. Lett. 83, 2116 (1999); G. Gabrielse et al., Phys. Rev. Lett. 82, 3198 (1999); R. Bluhm, V.A. Kostelecký, and N. Russell, Phys. Rev. Lett. 82, 2254 (1999); Phys. Rev. Lett. 79, 1432 (1997); Phys. Rev. D 57, 3932 (1998); D. Colladay and V.A. Kostelecký, Phys. Lett. B 511, 209 (2001); G.M. Shore, Nucl. Phys. B 717, 86 (2005).

[19] F.W. Stecker and S.L. Glashow, Astropart. Phys. 16, 97 (2001); B. Altschul, Phys. Rev. D 74, 083003 (2006); Astropart. Phys. 28, 380 (2007).

[20] B.R. Heckel et al., Phys. Rev. Lett. 97, 021603 (2006); L.-S. Hou, W.-T. Ni, and Y.-C.M. Li, Phys. Rev. Lett. 90, 201101 (2003); R. Bluhm and V.A. Kostelecký, Phys. Rev. Lett. 84, 1381 (2000); H. Müller, S. Herrmann, A. Saenz, A. Peters, and C. Lammerzahl, Phys. Rev. D 70, 076004 (2004); H. Müller, Phys. Rev. D 71, 045004 (2005); B.R. Heckel et al., Phys. Rev. D 78, 092006 (2008).

[21] D. Bear, R.E. Stoner, R.L. Walsworth, V.A. Kostelecký, and C.D. Lane, Phys. Rev. Lett. 85, 5038 (2000); D.F. Phillips et al., Phys. Rev. D 63, 111101(R) (2001); M.A. Humphrey et al., Phys. Rev. A 68, 063807 (2003); F. Canè et al., Phys. Rev. Lett. 93, 230801 (2004); M. Romalis, in CPT and Lorentz Symmetry IV, edited by V.A Kostelecký (World Scientific, Singapore, 2008); V.A. Kostelecký and C.D. Lane, J. Math. Phys. 40, 6245 (1999); C.D. Lane, Phys. Rev. D 72, 016005 (2005); D. Colladay and P. McDonald, Phys. Rev. D 73, 105006 (2006).

[22] V.A. Kostelecký and C.D. Lane, Phys. Rev. D 60, 116010 (1999).

[23] P. Wolf, F. Chapelet, S. Bize, and A. Clairon, Phys. Rev. Lett. 96, 060801 (2006).

[24] R. Bluhm, V.A. Kostelecký, C.D. Lane, and N. Russell, Phys. Rev. Lett. 88, 090801 (2002); Phys. Rev. D 68, 125008 (2003).

[25] O. Bertolami et al., Phys. Lett. B 395, 178 (1997); G. Lambiase, Phys. Rev. D 72, 087702 (2005); J.M. Carmona et al., Mod. Phys. Lett. A21, 883 (2006).

[26] H. Nguyen (KTeV Collaboration), arXiv:hep-ex/0112046; A. Di Domenico (KLOE Collaboration), in CPT and Lorentz Symmetry $I V$, edited by V.A. Kostelecký (World Scientific, Singapore, 2008); R. Ackerstaff et al.(OPAL Collaboration), Z. Phys. C 76, 401 (1997); M. Feindt et al.(DELPHI Collaboration), preprint DELPHI 97-98 CONF 80 (1997); K. Abe et al.(BELLE Collaboration), Phys. Rev. Lett. 86, 3228 (2001); B. Aubert et al.(BaBar Collaboration), Phys. Rev. Lett. 92, 142002 (2004); Phys. Rev. Lett. 100, 131802 (2008); J.M. Link et al.(FOCUS Collaboration), Phys. Lett. B 556, 7 (2003); V.A. Kostelecký, Phys. Rev. Lett. 80, 1818 (1998); Phys. Rev. D 61, 016002 (1999); Phys. Rev. D 64, 076001 (2001); N. Isgur et al., Phys. Lett. B 515, 333 (2001).

[27] G.W. Bennett et al.( $g-2$ Collaboration), Phys. Rev. Lett. 100, 091602 (2008); V.W. Hughes et al., Phys. Rev. Lett. 87, 111804 (2001); R. Bluhm, V.A. Kostelecký and C.D. Lane, Phys. Rev. Lett. 84, 1098 (2000).

[28] L.B. Auerbach et al.(LSND Collaboration), Phys. Rev. D 72, 076004 (2005); M.D. Messier (SK), in CPT and Lorentz Symmetry III, edited by V.A. Kostelecký (World 
Scientific, Singapore, 2005); P. Adamson et al. (MINOS Collaboration), Phys. Rev. Lett. 101, 151601 (2008); V.A. Kostelecký and M. Mewes, Phys. Rev. D 69, 016005 (2004); Phys. Rev. D 70, 031902(R) (2004); Phys. Rev. D 70, 076002 (2004); T. Katori, V.A. Kostelecký, and R. Tayloe, Phys. Rev. D 74, 105009 (2006); V. Barger, D. Marfatia, and K. Whisnant, Phys. Lett. B 653, 267 (2007).

[29] D.L. Anderson, M. Sher, and I. Turan, Phys. Rev. D 70, 016001 (2004); E.O. Iltan, Mod. Phys. Lett. A 19, 327 (2004).

[30] J.B.R. Battat, J.F. Chandler, and C.W. Stubbs, Phys. Rev. Lett. 99, 241103 (2007); H. Müller, S.W. Chiow, S. Herrmann, S. Chu, and K.Y. Chung, Phys. Rev. Lett. 100, 031101 (2008); W.M. Jensen, S.M. Lewis, and J.C. Long, in CPT and Lorentz Symmetry IV, edited by V.A. Kostelecký (World Scientific, Singapore, 2008).

[31] Q.G. Bailey and V.A. Kostelecký, Phys. Rev. D 74, 045001 (2006); Q.G. Bailey, arXiv:0904.0278 [gr-qc].

[32] M.A. Hohensee, R. Lehnert, D.F. Phillips, and R.L. Walsworth, Phys. Rev. Lett. 102, 170402 (2009).

[33] V.A. Kostelecký and M. Mewes, Phys. Rev. Lett. 97, 140401 (2006).

[34] S. Herrmann et al., in CPT and Lorentz Symmetry IV, edited by V.A. Kostelecký (World Scientific, Singapore, 2008).

[35] M.A. Hohensee, P.L. Stanwix, M.E. Tobar, D.F. Phillips, and R.L. Walsworth, to be published.

[36] S.R. Coleman and S.L. Glashow, Phys. Rev. D 59, 116008 (1999).

[37] See, e.g., E.F. Beall, Phys. Rev. D 1, 961 (1970); T. Jacobson, S. Liberati, and D. Mattingly, Phys. Rev. D 67, 124011 (2003).

[38] R. Lehnert, Phys. Rev. D 68, 085003 (2003).

[39] R. Lehnert and R. Potting, Phys. Rev. Lett. 93, 110402 (2004); Phys. Rev. D 70, 125010 (2004) [Erratum-ibid. D 70, 129906 (2004)].

[40] B.D. Altschul, Nucl. Phys. B 796, 262 (2008).

[41] R. Assmann et al. (LEP Energy Working Group), Eur. Phys. J. C 39, 253 (2005).

[42] The analysis in Ref. [16] implicitly scales the coordinates such that $c_{p}^{\mu \nu}=0$.

[43] In fact, in Appendix A we recall that tests with Csfountain clocks bound the eight anisotropic components of $2 c_{p}^{\mu \nu}-\tilde{k}^{\mu \nu}$ with sensitivities of $10^{-21} \ldots 10^{-25}$, so that some of these eight components can actually be dropped from the UHECR analysis in Ref. [16].
[44] This does not necessarily entail violations of causality [45]. For example, we can reinterpret the model employing the coordinate rescaling discussed in Sec. III In the rescaled Minkowski spacetime, photons moves on the new light cone; fermions, which now exhibit $c^{\mu \nu}$ Lorentz violation, have a maximal speed less than that of the photon. Thus, both the photon and the fermion obey conventional causality with respect to the new spacetime.

[45] V.A. Kostelecký and R. Lehnert, Phys. Rev. D 63, 065008 (2001); C. Adam and F.R. Klinkhamer, Nucl. Phys. B 607, 247 (2001).

[46] During the final stages of preparing this manuscript for publication, another preprint studying photon decay in the $\tilde{\kappa}_{\text {tr }}$ model appeared on the arXiv: F.R. Klinkhamer and M. Schreck, Phys. Rev. D 78, 085026 (2008).

[47] F. Aharonian et al. (The HEGRA Collaboration), Astrophys. J. 614, 897 (2004).

[48] K. Ackerstaff et al. (OPAL Collaboration), Eur. Phys. J. C 2, 39 (1998).

[49] P. Achard et al. (L3 Collaboration), Phys. Lett. B 531, 28 (2002).

[50] G. Abbiendi et al. (OPAL Collaboration), Eur. Phys. J. C 26, 331 (2003).

[51] V.M. Abazov et al. (D0 Collaboration), Phys. Lett. B 639, 151 (2006) [Erratum-ibid. B 658, 285(E) (2008)].

[52] V.M. Abazov et al. (D0 Collaboration), Phys. Lett. B 666, 435 (2008).

[53] S. Abachi et al. (D0 Collaboration), Nucl. Instrum. Meth. A 338, 185 (1994).

[54] V.A. Kostelecký and A.G.M. Pickering, Phys. Rev. Lett. 91, 031801 (2003).

[55] B. Altschul, arXiv:0905.4346.

[56] Q.G. Bailey and V.A. Kostelecký, Phys. Rev. D 70, 076006 (2004).

[57] H. Müller, Phys. Rev. D 71, 045004 (2005).

[58] C. Adam and F.R. Klinkhamer, Nucl. Phys. B 657, 214 (2003).

[59] D. Colladay and V.A. Kostelecký, Phys. Lett. B 511, 209 (2001).

[60] R. Lehnert, J. Math. Phys. 45, 3399 (2004).

[61] In the present case, we have selected the Sun-centered celestial equatorial frame. Since the $\tilde{\kappa}_{\text {tr }}$ term is isotropic, Lagrangian (B4) also holds in all inertial Minkowski coordinate systems at rest relative to the Sun-centered celestial equatorial frame. 